\title{
Exploration of reproductive healthcare needs among adult men regarding sexual transmitted diseases and HIV/AIDS
}

\author{
Leila Karimi ${ }^{1}$, Khadijeh Mirzaii Najmabadi ${ }^{2}$, Abbas Ebadi $^{3}$, Asiyeh Pormehr-Yabandeh ${ }^{4}$
}

${ }^{1}$ Ph.D. Candidate of Reproductive Health, Department of Midwifery, Faculty of Nursing and Midwifery, Mashhad University of Medical Sciences Mashhad, Iran

${ }^{2}$ Associate Professor, Department of Midwifery, Faculty of Nursing and Midwifery, Mashhad University of Medical Sciences Mashhad, Iran

${ }^{3}$ Associate Professor, Ph.D. of Nursing, Behavioral Sciences Research Center, Nursing Faculty, Baqiyatallah University of Medical Sciences, Tehran, Iran

${ }^{4}$ M.Sc. of Midwifery, Mother and Child Welfare Research Center, Department of Midwifery, Hormozgan University of Medical Sciences, Bandar Abbas, Iran

\section{Type of article: Original}

\begin{abstract}
Introduction: One of the most important public health issues is men's sexual and reproductive health. Men are the most important counterparts in the prevention of sexual transmitted diseases and HIV/AIDS. A low level of men's referral to utilize healthcare services and the stigmatization of reporting sexually transmitted diseases in society due to cultural issues calls for the designation of strategies for improving the men's healthcare conditions. In addition, it is required to assess men's healthcare needs with consideration of cultural-contextual, social, and economic issues. The aim of this study was to explore the reproductive healthcare needs of men regarding sexual transmitted diseases and HIV/AIDS.

Methods: This was a qualitative study with 34 men and six women from the general population; health policymakers, reproductive health providers, and clergies were collected in two large cities of Iran, including Tehran and Mashhad, in 2015 through individual in-depth interviews. Participants were chosen through purposive sampling. In-depth individual interviews were conducted to collect data. All interviews were transcribed verbatim and using data analysis through conventional qualitative content analysis. Data were analyzed using conventional qualitative content analysis with MAXqda.

Results: The data analysis resulted in the development of four themes and 10 categories. The themes were "men's educational empowerment," "appropriate sociocultural background with advocacy," "organizing sexual needs based on sexual ethics, religious doctrine and women's empowerment," and "meeting men's preventive, caring and welfare needs."

Conclusion: Because men's reproductive health is intertwined with public health, data collected regarding men's healthcare needs with the consideration of social and cultural factors can be used for designing strategies for reducing the incidence/prevalence rates of STDs and HIV/AIDS.
\end{abstract}

Keywords: Men, Needs, Reproductive healthcare, Sexual transmitted diseases, AIDS

\section{Introduction}

Half of the active population of every society and the pillar of the family are men (1). Men's health has cultural and social implications because a significant portion of every country's businesses is run by men. Therefore, any increase in a country's economic growth depends on men's mental and physical health. Therefore, men's health is considered one the most important affairs of both the family and society. Therefore, the field of men's health is

\section{Corresponding author:}

Associate Professor Dr. Khadijeh Mirzaii Najmabadi, Department of Midwifery, Faculty of Nursing and Midwifery, Mashhad University of Medical Sciences, Mashhad, IR Iran.

Tel: +98.5138598016, Fax: +98.5138598016, E-mail: erfan1383@gmail.com

Received: August 09, 2016, Accepted: November 21, 2016, Published: September 2017

iThenticate screening: October 02, 2016, English editing: December 22, 2016, Quality control: June 17, 2017

(C) 2017 The Authors. This is an open access article under the terms of the Creative Commons Attribution-NonCommercialNoDerivs License, which permits use and distribution in any medium, provided the original work is properly cited, the use is non-commercial and no modifications or adaptations are made. 
typically expanded upon, and various countries are planning to improve the health of this important portion of society $(2,3)$. One the most important aims of reproductive health improvement programs is the prevention and management of sexual transmitted diseases (STDs) in the healthcare system Also, this study aims to increase the attention of public and religious leaders as well as policymakers to the prevalence of HIV in societies (4). World leaders at the World Summit in New York (2005) emphasized the necessity of placing sexual equality, HIV/AIDS, and reproductive healthcare on the agenda $(5,6)$. In the International Conference on Population and Development in Cairo (1994), the provision of education and health, including reproductive healthcare needs, was announced as the requirement of sustainable development. In addition, the aim of reducing the incidence/prevalence rate of HIV/AIDS was agreed upon by participating countries (3). Statistics show that, in $2013,11 \%$ of women and $89 \%$ of men were diagnosed with HIV/AIDS. Also, 33\% of the causes of HIV/AIDS transmission were the result of unsafe sexual relationships with a prevalence rate of $67.5 \%$ and $32.5 \%$ for men and women, respectively (7). If the prevalence of STDs is increased, the prevalence rate of HIV/AIDS infection mounts, and the risk of HIV virus infection is increased six to 10 times (8). Taking into account the portion of men in society and the importance of the workforce in the development of social economy (9), they become the most important counterparts in the prevention of sexual transmitted diseases and HIV/AIDS (10). Nevertheless, a low level of men's referral to utilize healthcare services and the stigmatization of the STDs reported in society due to cultural issues calls for the designation of strategies for improving men's healthcare conditions. In the first step, a need assessment is required in order to provide information with regard to the health condition and needs intertwined with cultural-contextual, social, and economic issues (11). There has been a lack of knowledge on men's healthcare needs regarding STDs and HIV/AIDS. In addition, the majority of studies in the field of reproductive healthcare focused on women who were in the reproductive age (12). Therefore, men's reproductive healthcare needs were mainly ignored or focused on educational needs with a quantitative approach (13). The aim of this study was to explore the reproductive healthcare needs of adult men regarding sexual transmitted diseases and HIV/AIDS.

\section{Material and Methods}

This study was conducted between March and October 2015. The participants consisted of 34 men and six women chosen using a purposeful sampling method. A qualitative design using a content analysis approach was conducted. The reason for the selection of a qualitative design to study this phenomenon was the unknown identity of men's needs, the influence of culture and context on the development and presentation of their needs, and inability of the quantitative design to study phenomena with a complex and unknown nature (14). Of the participants, 17 people were ordinary citizens (general population), 19 were expert participants in the field of the study, including medical specialists (dermatologist, infectious diseases' specialist, gynecologist, and urologist), healthcare providers, clerics, and healthcare policy makers, and four men suffering from HIV/AIDS. All participants were chosen from Tehran and Mashhad in Iran. The maximum variation in sampling was considered in terms of age, marital status, employment, educational level, and the socio-economic-cultural status. The inclusion criteria for ordinary people were age above 18 years old, being Iranian and able to talk in Farsi, no history of psychological disorders, and willingness to participate in this study. Also, having the experience of assessment, consultation, and treatment of men with STDs and HIV/AIDS and willingness to be interviewed were the inclusion criteria to choose the specialists. Semistructured interviews were conducted by the first author LK to gather data. The participants determined the time and place for interviews convenient to them. The interviews took place in hospitals, physician clinics, and the participants' own homes. The focus of the questions asked during the interviews were: 1) What are your needs regarding reproductive healthcare? 2) Will you please share your experiences of STDs and HIV/AIDS? Probing questions were asked to follow the participants' perspectives and increase the depth of the interviews (15). Each person was interviewed once, which lasted on average between 30 and 60 minutes. The data collection was continued until the researchers reached data saturation (16). It meant that no new data were collected to add to the variations of the developed themes and categories. This study research proposal was approved by the research council affiliated with Mashhad University of Medical Sciences, Mashhad, Iran, which corroborated its ethical considerations (decree number: 932914). The participants were informed of the purpose and method of the study. They also were informed that participation was voluntary and anonymous, and they could withdraw from the study at any time. Also, they were ensured of the confidentiality of data, and permission to tape record the interviews was obtained. Conventional content analysis was used to analyze data. The interviews and the researchers' observations of the participants' non-verbal communication were transcribed verbatim. They were read several times to obtain the sense of whole. After determining the unit of analysis and meaning units, the transcriptions were coded. The MAXQDA v.10 software was applied to manage data. The extracted codes were compared together and classified in terms of similarities and differences to develop categories. Next, themes were explored through the development of the relationships between the categories (17). Procedures used in this study for rigor were listening to the interviews 
and reading the transcriptions for several times, asking the perspectives of two external peers on the data analysis process, and incorporating their feedback into findings, and the maximum variation in sampling. Also, external auditing by a team of experts was conducted to check the compatibility between the study aim, analysis process, and findings (Guba \& Lincoln, 1985). For member checking, a brief report of the findings was provided to a couple of men who were asked to confirm that we reflected upon their perspectives and comments. Also, we tried to improve the transferability of our findings through the provision of details of the study process and the participants' direct quotations (18).

\section{Results}

\subsection{General findings}

Mean age of participants was 33.5 years $(\mathrm{SD}=9.085)$ with a range from 19 to 66 years old. The majority of patients had a bachelor (60.7\%) and higher academic degree. They were married and their work experiences $(58.5 \%)$ were more than 10 years old. The data analysis was resulted in the development of four themes and 10 categories (Table1). They were presented below using the direct quotations of the participants.

Table 1. Reproductive healthcare needs of men with regard to HIV/AIDS

\begin{tabular}{|l|l|}
\hline Category & Theme \\
\hline $\begin{array}{l}\text { Necessity of informing and education; necessity of empowerment with a } \\
\text { gender approach; necessity of attention to educational appropriate } \\
\text { characteristics }\end{array}$ & Men's educational empowerment \\
\hline $\begin{array}{l}\text { Culture making in accordance with the religious doctrine, Islam, and the } \\
\text { culture of Iran; attracting string support }\end{array}$ & $\begin{array}{l}\text { Appropriate sociocultural } \\
\text { background with advocacy }\end{array}$ \\
\hline $\begin{array}{l}\text { Necessity of guided and restrained efficiency; sexual instinct from Islam's } \\
\text { perspective; the need for effective management and education of skills to } \\
\text { control sexual relationships; the need for comprehensive support of women }\end{array}$ & $\begin{array}{l}\text { Organizing sexual needs based on } \\
\text { sexual ethic, religious doctrine, and } \\
\text { women's empowerment }\end{array}$ \\
\hline $\begin{array}{l}\text { The need to adopt caring-preventive approaches; meeting services and } \\
\text { welfare needs }\end{array}$ & $\begin{array}{l}\text { Meeting preventive, caring and } \\
\text { welfare needs }\end{array}$ \\
\hline
\end{tabular}

\subsection{Men's educational empowerment}

The necessity of improving men's knowledge and education about STDs and HIV/AIDs were emphasized in this study. Participants complained about receiving little and nonsystematic information about STDs and HIV/AIDs by healthcare professionals. In addition, the information was scarce, lacked sufficient details, and only provided in special days of the year such as "the AIDS week" or "Men's Day." "Men need to receive appropriate and reliable information about AIDS and STDs. The information should be provided honestly and with details. However, it does not happen in many cases. For instance, the information is evaded, and the details of education are censured" (policymaker, 55 years old, male). A lack of provision of learning situations and educational facilities such as appropriate place and time made the men's education challenging. For instance, the participants had not enough time to attend the educational sessions due to busy workdays. They stated that the inability of policymakers in the healthcare system to organize an appropriate time and place for men's education showed that men's reproductive healthcare needs were not considered a priority. "I am afraid, the education provided to men is delivered in the morning, and as you know I go to work from the early morning to evening. Therefore, I have no time to attend the educational sessions" (bank employee, 52 years old, male). "... Where should I receive the education? Please determine the time and place for me over the week to be able to describe my problems to a cognate healthcare provider" (engineer, 38 years old, male). From the participants' perspectives, because men had no sufficient information about STDs' transmission mechanisms, they could not consider themselves vulnerable to such diseases. “... These diseases [STDs and HIV/AIDS] belong to the low-class portion of the society. I belong to a good family and do nothing wrong to make me sick. Why should I be stricken by such diseases? I have made no sin. I am not scared of these diseases at all" (construction worker, 34 years old, male). According to the participants, an appropriate designation of the educational program, provision of appropriate facilities, and improvement of their motivation led to better understanding of the mechanisms of these diseases. Also, sensitizing the participants to the importance of STDs and HIV/AIDS would lead to effective education. "Men scarcely feel that they need education about reproductive health care. They should be sensitized. They should be informed about the fact that they might be stricken by these diseases. They need to know about the complications of these diseases influencing both the person and family and also routes of transmission. They should be informed that they are in danger and that high-risk persons need emergency care" (specialist in the field of infectious diseases, 36 years old, male. Participants emphasized the need for receiving skill-based education with a focus on sexual issues. "Adolescents and young 
adults need information. They need such information to face related issues in the society. They should be armed with practical strategies to be able to prevent from such diseases" (specialist in the field of social medicine, 54 years old, male). It was stated that any educational program would not lead to effective learning. The characteristics of the appropriate educational program that could lead to optimum learning were those planned based on men's needs and age conditions, were systematic and practical, compatible with social values, religious beliefs, and culture. Some participants believed that reproductive healthcare education for men were required to be mandatory and should be started from schools. "...education should be incorporated into textbooks and courses for girls and boys. Also, attractive educational methods should be used, and a final examination should be taken to ensure of students' learning" (general physician, 34 years old, male). "Men are mostly busy with work and even have no free time until night ... Local mosques and cultural centers close to men's work place can be used as men refer to those places time to time and have no worries for losing their work times" (local clinic administrator, male). Other participants believed in the necessity of media and educational-cultural collaboration to improve men's reproductive healthcare conditions. "Media, including newspapers, radio, and television, should be involved in education. When a man is unable to attend educational sessions, he can receive education delivered by media" (retired employee of the ministry of education, 66 years old, male). A cleric participant emphasized the importance of education of men regarding reproductive healthcare and sexual issues using religious doctrine. "Education regarding sexual issues should be conducted by cognate persons from the same gender because this is a private matter, and some men are unable to discuss about them with people from the opposite gender. I believe a male educator should educate a man" (cleric, faculty member, 62 years old, male). "For education, people can use religious and Islamic doctrine because Islam has the answer to all questions" (the same participant).

\subsection{Appropriate sociocultural background with Advocacy}

From the participants' perspectives, there is a need for culture-making in society with regard to STDs and AIDS/HIV. Also, the roles of religion and culture as the cornerstones of culture-making have been emphasized. "Stigmatization of people with STDs and HIV/AIDS and the negative attitudes of people toward these diseases and wrong perceptions are barriers to reporting these diseases because many patients are afraid of being rejected by society. Therefore, the incident rate of these diseases is growing day by day" (administrator of consultation center, 48 years old, male). The relationship between STDs and HIV/AIDS and stigmatization of patients hinder direct public education and education by media. "One important issue is the impossibility of education about STDs and even the prevention of HIV/AIDS through using radio and television. For instance, the use of condoms is never discussed publicly" ( $\mathrm{PhD}$ in the field of health education, 29 years old, male). From the participants' perspectives, changes in social values, information technology, and satellite programs have brought many changes to the public lifestyle. For instance, marriage was delayed, and a time gap was created between the adolescence period and marriage time. In addition, sexual needs were influenced by Western societies' ideals and advertisements, which have resulted in the development of high-risk sexual relationships, STDs, and HIV/AIDS. "Some satellite programs advertise offbeat sexual relationships. Those adolescents who have no information about sexual issues may be influenced by such programs and get inclined to practice sexual relationships" (military, 36 years old, male). For the successful implementation of any programs aiming to reduce the prevalence of STDs and HIV/AIDS, policymakers and authorities should remove their silence and publicly speak about the burden of these diseases on society and design strategies to prevent them. Also, the participants suggested people's cooperation, a united collaboration between the ministry of health and other organizations and government support. In addition, the significance of marriage and family development support by the government through granting low fair loans for housing and marriage costs have been in line with strategies aimed to prevent STDs and HIV/AIDS. "We need to guide our young adults to get married because marriage can stabilize the family and help with the prevention of STDs and HIV/AIDS" (administrator of the social welfare institution, 49 years old, male). Some participants believed that young adults were not interested in listening to parent's advice. Therefore, visual education through video clips would be a replacement for face-to-face education for attracting your adults' attention. "The production of educational movies in which public figures such as athletes, actors, and well-known scientists being presented as the models of social ethical behavior providing education to young adults can lead to effective education. Also, such education can be provided in popular TV programs and shows" (policymaker, 47 years old, male).

\subsection{Organizing sexual needs based on sexual ethic, religious doctrine and women's empowerment}

The participants stated that Islam as a complete region has taken care about all aspects of the human being's life. Also, sexual derive as a physiologic need could be controlled and guide by ethical and religious principles to lead to the human being's ethical improvement and excellence. A cleric participants believed in the unavoidability of the sexual derive in the human being. From his perspective, the sexual derive should be controlled using wisdom, 
religious and ethical principles and strategies suggested by Islam. "According to the Islamic narratives, Chasity from the sexual derive should be sought. It means that the sexual derive should be controlled and restricted in the framework of wisdom, religion. Also, continence should be sought until the person gets married." (Cleric, male). The role of women in the prevention of STDs and HIV/AIDs was discussed. It was mentioned that the provision of required information to women especially to high risk ones could change their attitudes as well as their risky behaviors. Also, the women can be empowered to protect themselves and others against high risk situations that might lead to STDs and HIV/AIDS infection. "Women in our society are more vulnerable to STDs and HIV/AIDS than men. The causes of such vulnerability are economic, cultural and health issues. Some women are forced to surrender to unethical behaviors due to financial needs and social violence." (The gynecologist, 37 years old, female).

\subsection{Meeting preventive, caring, and welfare needs}

The pillar of prevention of STDs and HIV/AIDS is the identification of high risk people. While random screening in public and governmental places has been recognized as an appropriate strategy to identify risky people, it was mentioned that the majority of risky people were not employed by governmental settings. "There are a lot of patients, but no appropriate screening method is available to identify them. We cannot request diagnostic tests for all men. The screening equipment can be placed in some organizations and institutions. Perhaps it would be fine to request men to go under diagnostic tests once a year or every two years" (consultant in the AIDS prevention center, 54 years old, male). Preventive interventions were not confined to healthy people, but it encompassed the encouragement of real patients for referring to healthcare settings. "A short-term treatment process, short waiting time, and free treatment are required. It is also necessary to encourage patients to seek treatment and refer to the healthcare settings to follow-up their treatment" (neurologist, 59 years old, male). STDs and HIV/AIDS were not considered only medical diseases but also were social issues. Therefore, prevention and treatment of patients with these diseases needed more than medical treatment and required sociological and psychological interventions. "An inappropriate behavior with patients with STDs and HIV/AIDS results in public loss in terms of discouragement of patients' report of their diseases and referral to healthcare settings that increases the transmission of these diseases to others" (driver with HIV, 64 years old, male). One of the main social and welfare needs of men was the optimization of healthcare services' utilization in the society. "There is a need for facilities and equipment such as AIDS counseling centers before marriage, risk reduction centers, the provision of access to condom, syringe and sterile hand gloves for drug usage so on. The economic conditions of some people hinder access the facilities, also" (specialist in the field of skin and STDs, 49 years old, male). Participants called for the establishment of specialized hospitals and healthcare settings as well as consultation centers to provide appropriate care and treatment to men. Because the men were working in the morning shifts, the presence of healthcare staff in the centers during evening and night work shifts would facilitate men's referral to such settings.

\section{Discussion}

The findings of this study should be transferred with caution to other countries' healthcare systems with the consideration of cultural-contextual differences. This was the first qualitative study conducted on men's reproductive healthcare needs with regard to STDS and HIV/AIDs in Iran. The analyzed data led to the classification of the men's needs in four domains: educational, cultural, social, and sexual and caring. The majority of our study participants declared the necessity of the provision of clear information to men regarding STDs and HIV/AIDS. Because they had little knowledge about these diseases, they needed to learn about transmission routes and prevention methods. The participants' main areas of concern were a lack of guideline, appropriate place for the provision of education and care to men, being busy with work, an educator from the same gender, and the need for sexual issues education to adolescents in schools. According to our findings, the provision of education to men in mosques and cultural centers as well as gyms for facilitating education was suggested. Similarly, the findings of the study by Yam anis in Tanzania suggested that the provision of educational facilities in gyms and other public places for young adults who were deprived of formal education could reduce the prevalence of HIV/AIDS (19). Also, providing counselling and HIV screening tests in in the workplace and education about condom use to employed men in a transportation company led to behavior changes and the reduction of new infections incidences (11). According to the study by Oakley, not only was there a need for improvement of knowledge about STDs and HIV/AIDS, but also it was crucial to learn the skills required to use the knowledge in practice (20). In addition, Rahmati emphasized the need for learning life skills such as decision-making, problem solving, and the presence of a relationship between these skills and STDs and HIV/AIDS (21). In this study, the majority of men did not understand the risk for the transmission of STDs and HIV/AIDS and believed that it belonged to someone else. Similarly, Mohamadifar showed that $71.1 \%$ of students undermined the risk of AIDS transmission to them, though 
they followed high-risk behaviors such as unprotected sexual relationships and a history of STDs. In this respect, educational interventions can sensitize students about their vulnerability to STDs and HIV/AIDS and internalize risk reduction behaviors (5). In our current study, sensitizing before education using systematic education was recognized effective enough to help reduce high-risk behaviors. According to the participants' perspectives, highrisk behaviors, immigration, unemployment, a lack of health entertainment, a time gap between adolescence and marriage, divorce, and a large number of young adults were mentioned as the favorable causes of STDs and HIV/AIDS. The same results were reported in a study of Bagheri (22). In addition, the low status of women in sexual relationships and not being able to avoid high-risk behaviors made the empowerment of women necessary. Green stated that the risk for STDs and HIV transmission to women was high because women were unable to cut their sexual relationship due to financial and emotional needs and therefore were unable to advise their partner to use a condom (2). Generally, educational interventions, the provision of access to caring-preventive services, culture making, and patient support can prevent the transmission of STDs and HIV/AIDS. In Western societies, educational facilities are provided to children in the beginning of school age, but in the Iranian society such education are only provided informally due to cultural-contextual boundaries. In addition, unlike Western societies, failures in prevention methods such as condoms and syringes for drug use have led to the spread of STDs and HIV/AIDS.

\section{Conclusions}

Based on the findings, men feel the need for sexual and reproductive health; these needs include "educational empowerment," “advocacy," “organizing sexual needs based on sexual ethics," "women's empowerment," and "meeting men's preventive, caring and welfare needs." These needs were affected by socio-cultural components. If health policymakers pay attention to these issues, it is possible to implement effective programs for improving men's sexual health and reducing the incidence/prevalence rates of STDs and HIV/AIDS.

\section{Acknowledgments:}

This article was one part of the first authors' PhD dissertation (decree number: 931429) supported financially by Mashhad University of Medical Sciences, Mashhad, Iran. The researchers would like to express their gratitude to the participants, deputy of research, and the administrator of the diseases prevention and management department of Iranian Ministry of Health and Medical Education.

\section{Conflict of Interest:}

There is no conflict of interest to be declared.

\section{Authors' contributions:}

All authors contributed to this project and article equally. All authors read and approved the final manuscript.

\section{References:}

1) Population of the world, Available from: http://en.worldstat.info/world.

2) Greene ME, Biddlecom AE, Pulrwitz J, Wulf D, Bankole A, Sing S. Involving men in reproductive health: contributes to development, Background paper to the public choices, private decisions: sexual and reproductive health and the millennium development goals. in United Nation Millennium Development project. 2004.

3) The United Nations International Conference on Population and Development (ICPD) was held from 5-13 September 1994 in Cairo E.

4) UNFPA. Reproductive health wamaosael, ... noted, "The Millennium Development Goals, particularly the eradication of extreme ... child mortality; maternal health, including reproductive health; and HIV/AIDS.13. Avalaible From: http:/www.unfpa.org/public/home/sitemap/icpd/MDGs/MDGs-ICPD.

5) Mohammadi MR, Kalajabadi Farahani F, Bahonar A. Parents' Attitudes towards Adolescent Boy's Reproductive Health Needs and Practice in Tehran. Iran J Psychiatry. 2007; 2: 13-24.

6) World summit on the information society Geneva 2003-Tunis 2005 DW-GD-E, 12 December 2003, Declaration of Principles Building the Information Society: a global challenge in the new Millennium.

7) Iran HIV epidemiology. 2012; Available from: http://www.iranhiv.com/epidemiology_iran.htm.

8) The word health report 1998 Littc Avfa, report of the Director-General. World health organization [homepage on the internet]. Geneva: The organization; c1948-2005[updated 2004; cited 1998]. Available from: http://www.searo.who.int/rc52/rc51/rc51-inf-5.htm. 2005.

9) United nation Population fund (UNFPA), 2009. Available from: http://www.unfpa.org/intercenter/role4men/enhancin.htm. 
10) Ministry of Health. HIV/AIDS statistics. Tehran, Center for Disease Management. 2010.

11) Kaufman RA, English FW. Needs assessment: Concept and application. Educational Technology. 1979; 355.

12) Mbu RE, Takang WA, Fouedjio HJ, Joan E, Fouelifack FY, Tumasang FN, et al. Reproductive Health Needs of Women Living with HIV/AIDS in Yaounde, Cameroon. World Journal of AIDS. 2014; 4(1): 814. doi: 10.4236/wja.2014.41002.

13) Hajizadeh M, Javadnoori M, Javadifar N. Educational needs of adult men regarding sexual and reproductive health in Ahvaz, Iran. Journal of Midwifery and Reproductive Health. 2015; 3(3): 385-93. doi: 10.22038/jmrh.2015.4314.

14) Holloway I, Wheeler S. Qualitative Research in Nursing and Healthcare, 3rd Edition. 2010; 368.

15) Streubert SH, Carpenter D. Qualitative research in nursing. 4 ed. Philadelphia: Lippincott Williams \& Wilkins. 2007.

16) Adib Hajbagherie M, Parvizie S, Salsalie M. Qualitative research methods. 2 ed. Tehran: Boshra Publisher; 2010.

17) Elo S, Kyngas H. The qualitative content analysis process. J Adv Nurs. 2008; 62(1): 107-15. doi: 10.1111/j.1365-2648.2007.04569.x. PMID: 18352969.

18) Polit DF, Beck CT. Nursing Research: Generating and Assessing Evidence for Nursing Practice. 2006.

19) Yamanis TJ, Maman S, Mbwambo JK, Earp JA, Kajula LJ. Social venues that protect against and promote HIV risk for young men in Dar es Salaam, Tanzania. Soc Sci Med. 2010; 71(9): 1601-9. doi: 10.1016/j.socscimed.2010.07.039. PMID: 20846768, PMCID: PMC2952658.

20) Oakley A, Fullerton D, Holland J, Arnold S, France-Dawson M, Kelley P, et al. Sexual health education interventions for young people: a methodological review. Bmj. 1995; 310(6973): 158-62. doi: 10.1136/bmj.310.6973.158. PMID: 7833754, PMCID: PMC2548556.

21) Rahmati Najarkolaei F, Khoobdel M, Ghasemi M, Farmanbar R, Jafari MR, Khamalikhah T. Knowledge and high risk behaviors of newly intered student to Tehran University toward HIV/AIDS. Holistic nursing and midwifery (The journal of nursing and midwifery faculties guilan medical university. 2013; 23(2(70)): 35-44.

22) Bagheri P, Faramarz H, Moshki M. The Use of Rural Health Centers as Rural Education Centers: A New Direction for Medical Education in Developing Countries. Thrita. 2013; 2(1): 38-9. doi: 10.5812/thrita.9032. 